\title{
Improving Semantic Composition with Offset Inference
}

\author{
Thomas Kober, Julie Weeds, Jeremy Reffin and David Weir \\ TAG laboratory, Department of Informatics, University of Sussex \\ Brighton, BN1 9RH, UK \\ \{t.kober, j.e.weeds, j.p.reffin, d.j.weir\}@sussex.ac.uk
}

\begin{abstract}
Count-based distributional semantic models suffer from sparsity due to unobserved but plausible co-occurrences in any text collection. This problem is amplified for models like Anchored Packed Trees (APTs), that take the grammatical type of a co-occurrence into account. We therefore introduce a novel form of distributional inference that exploits the rich type structure in APTs and infers missing data by the same mechanism that is used for semantic composition.
\end{abstract}

\section{Introduction}

Anchored Packed Trees (APTs) is a recently proposed approach to distributional semantics that takes distributional composition to be a process of lexeme contextualisation (Weir et al., 2016). A lexeme's meaning, characterised as knowledge concerning co-occurrences involving that lexeme, is represented with a higher-order dependencytyped structure (the APT) where paths associated with higher-order dependencies connect vertices associated with weighted lexeme multisets. The central innovation in the compositional theory is that the APT's type structure enables the precise alignment of the semantic representation of each of the lexemes being composed. Like other countbased distributional spaces, however, it is prone to considerable data sparsity, caused by not observing all plausible co-occurrences in the given data. Recently, Kober et al. (2016) introduced a simple unsupervised algorithm to infer missing cooccurrence information by leveraging the distributional neighbourhood and ease the sparsity effect in count-based models.

In this paper, we generalise distributional inference (DI) in APTs and show how precisely the same mechanism that was introduced to support distributional composition, namely "offsetting" APT representations, gives rise to a novel form of distributional inference, allowing us to infer co-occurrences from neighbours of these representations. For example, by transforming a representation of white to a representation of "things that can be white", inference of unobserved, but plausible, co-occurrences can be based on finding near neighbours (which will be nouns) of the "things that can be white" structure. This furthermore exposes an interesting connection between distributional inference and distributional composition. Our method is unsupervised and maintains the intrinsic interpretability of APTs ${ }^{1}$.

\section{Offset Representations}

The basis of how composition is modelled in the APT framework is the way that the co-occurrences are structured. In characterising the distributional semantics of some lexeme $w$, rather than just recording a co-occurrence between $w$ and $w^{\prime}$ within some context window, we follow Padó and Lapata (2007) and record the dependency path from $w$ to $w^{\prime}$. This syntagmatic structure makes it possible to appropriately offset the semantic representations of each of the lexemes being composed in some phrase. For example many nouns will have distributional features starting with the type amod, which cannot be observed for adjectives or verbs. Thus, when composing the adjective white with the noun clothes, the feature spaces of the two lexemes need to be aligned first. This can be achieved by offsetting one of the constituents, which we will explain in more detail in this section.

We will make use of the following nota-

\footnotetext{
${ }^{1}$ We release our code and data at https://github. com/tttthomassss/acl2017
} 
tion throughout this work. A typed distributional feature consists of a path and a lexeme such as in amod:white. Inverse paths are denoted by a horizontal bar above the dependency relation such as in $\overline{\text { dobj }}$ :prefer and higherorder paths are separated by a dot such as in $\overline{\text { amod. }}$. compound :dress.

Offset representations are the central component in the composition process in the APT framework. Figure 1 shows the APT representations for the adjective white (left) and the APT for the noun clothes (right), as might have been observed in a text collection. Each node holds a multiset of lexemes and the anchor of an APT reflects the current perspective of a lexeme at the given node. An offset representation can be created by shifting the anchor along a given path. For example the lexeme white is at the same node as other adjectives such as black and clean, whereas nouns such as shoes or noise are typically reached via the $\overline{\text { amod }}$ edge.

Offsetting in APTs only involves a change in the anchor, the underlying structure remains unchanged. By offsetting the lexeme white by amod the anchor is shifted along the $\overline{\text { amod }}$ edge, which results in creating a noun view for the adjective white. We denote the offset view of a lexeme for a given path by superscripting the offset path, for example the amod offset of the adjective white is denoted as white $^{\text {amod. }}$. The offsetting procedure changes the starting points of the paths as visible in Figure 1 between the anchors for white and white ${ }^{\text {amod }}$, since paths always begin at the anchor. The red dashed line in Figure 1 reflects that anchor shift. The lexeme white amod represents a prototypical "white thing", that is, a noun that has been modified by the adjective white. We note that all edges in the APT space are bi-directional as exemplified in the coloured amod and $\overline{\text { amod }}$ edges in the APT for white, however for brevity we only show uni-directional edges in Figure 1.

By considering the APT representations for the lexemes white and clothes in Figure 1, it becomes apparent that lexemes with different parts of speech are located in different areas of the semantic space. If we want to compose the adjective-noun phrase white clothes, we need to offset one of the two constituents to align the feature spaces in order to leverage their distributional commonalities. This can be achieved by either creating a noun offset view of white, by shift- ing the anchor along the $\overline{\text { amod }}$ edge, or by creating an adjective offset representation of clothes by shifting its anchor along amod. In this work we follow Weir et al. (2016) and always offset the dependent in a given relation. Table 1 shows a subset of the features of Figure 1 as would be represented in a vectorised APT. Vectorising the whole APT lexicon results in a very highdimensional and sparse typed distributional space. The features for white amod (middle column) highlight the change in feature space caused by offsetting the adjective white. The features of the offset view white ${ }^{\text {amod }}$, are now aligned with the noun clothes such that the two can be composed. Composition can be performed by either selecting the union or intersection of the aligned features.

\begin{tabular}{|c|c|c|}
\hline white & white $^{\text {amod }}$ & clothes \\
\hline :clean & amod:clean & amod:wet \\
\hline$\overline{\text { amod }}$ : shoes & : shoes & :dress \\
\hline$\overline{\text { amod. }} \cdot \overline{\text { dobj }}$ : wear & $\overline{\text { dobj: wear }}$ & 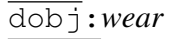 \\
\hline$\overline{\operatorname{amod}} \cdot \overline{n s u b j} \cdot e a r n$ & 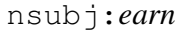 & $\overline{\mathrm{nsubj}}$ : admit \\
\hline
\end{tabular}

Table 1: Sample of vectorised features for the APTs shown in Figure 1. Offsetting white by amod creates an offset view, white $^{\text {amod }}$, representing a noun, and has the consequence of aligning the feature space with clothes.

\subsection{Qualitative Analysis of Offset Representations}

Any offset view of a lexeme is behaviourally identical to a "normal" lexeme. It has an associated part of speech, a distributional representation which locates it in semantic space, and we can find neighbours for it in the same way that we find neighbours for any other lexeme. In this way, a single APT data structure is able to provide many different views of any given lexeme. These views reflect the different ways in which the lexeme is used. For example law $\overline{w^{\text {nsubj }}}$ is the $\overline{\text { nsubj } j}$ offset representation of the noun law. This lexeme is a verb and represents an action carried out by the law. This contrasts with $l a w^{\overline{\text { dobj }}}$, which is the $\overline{\mathrm{dob} j}$ offset representation of the noun law. It is also a verb, however represents actions done to the law. Table 2 lists the 10 nearest neighbours for a number of lexemes, offset by amod, $\overline{\operatorname{dob} j}$ and

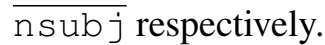

For example, the neighbourhood of the lexeme ancient in Table 2 shows that the offset view for ancient $t^{\text {amod }}$ is a prototypical representation of an "ancient thing", with neighbours easily associated with the property ancient. Furthermore, Table 2 


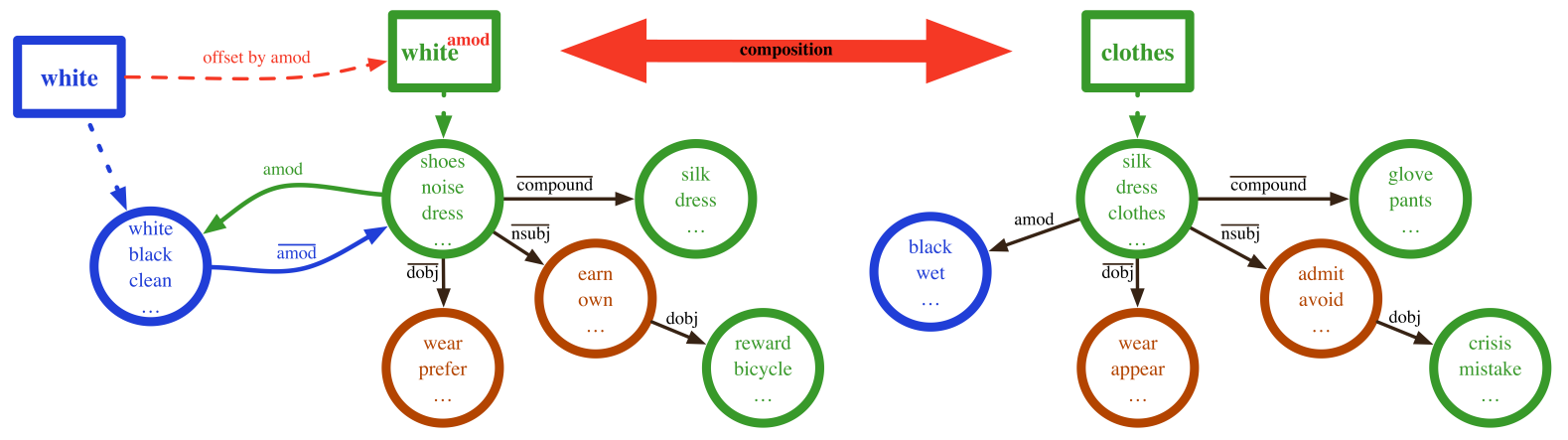

Figure 1: Structured distributional APT space. Different colours reflect different parts of speech. Boxes denote the current anchor of the APT, circles represent nodes in the APT space, holding lexemes, and edges represent their relationship within the space.

illustrates that nearest neighbours of offset views are often other offset representations. This means that for example actions carried out by a mother tend to be similar to actions carried out by a father or a parent.

\subsection{Offset Inference}

Our approach generalises the unsupervised algorithm proposed by Kober et al. (2016), henceforth "standard DI", as a method for inferring missing knowledge into an APT representation. Rather than simply inferring potentially plausible, but unobserved co-occurrences from near distributional neighbours, inferences can be made involving offset APTs. For example, the adjective white can be offset so that it represents a noun - a prototypical "white thing". This allows inferring plausible co-occurrences from other "things that can be white", such as shoes or shirts. Our algorithm therefore reflects the contextualised use of a word. This has the advantage of being able to make flexible and fine grained distinctions in the inference process. For example if the noun law is used as a subject, our algorithm allows inferring plausible co-occurrences from "other actions carried out by the law". This contrasts the use of law as an object, where offset inference is able to find cooccurrences on the basis of "other actions done to the law". This is a crucial advantage over the method of Kober et al. (2016) which only supports inference on uncontextualised lexemes.

A sketch of how offset inference for a lexeme $w$ works is shown in Algorithm 1. Our algorithm requires a distributional model $M$, an APT representation for the lexeme $w$ for which to perform offset inference, a dependency path $p$, describing the offset for $w$, and the number of neighbours $k$.
The offset representation of $w^{\prime}$ is then enriched with the information from its distributional neighbours by some merge function. We note that if the offset path $p$ is the empty path, we would recover the algorithm presented by Kober et al. (2016). Our algorithm is unsupervised, and agnostic to the input distributional model and the neighbour retrieval function.

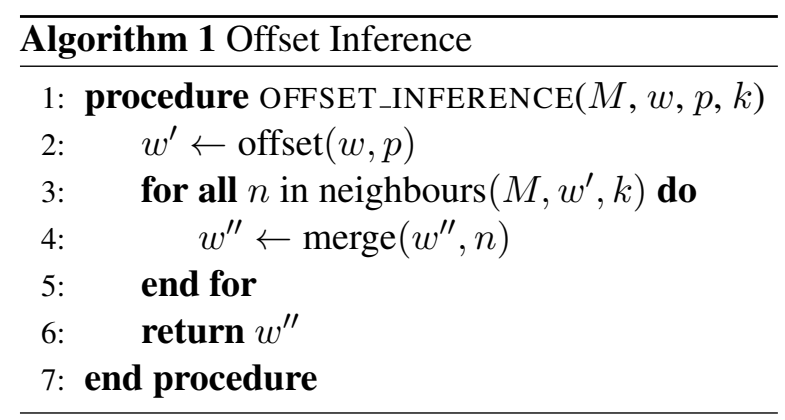

\section{Connection to Distributional Composition}

An interesting observation is the similarity between distributional inference and distributional composition, as both operations are realised by the same mechanism - an offset followed by inferring plausible co-occurrence counts for a single lexeme in the case of distributional inference, or for a phrase in the case of composition. The merging of co-occurrence dimensions for distributional inference can also be any of the operations commonly used for distributional composition such as pointwise minimum, maximum, addition or multiplication.

This relation creates an interesting dynamic between distributional inference and composition when used in a complementary manner as in this work. The former can be used as a process of $\mathrm{co}$ occurrence embellishment which is adding miss- 


\begin{tabular}{|c|c|}
\hline Offset Representation & earest Neighbours \\
\hline $\begin{array}{l}\text { ancient }^{\text {amod }} \\
\text { red }^{\text {amod }} \\
\text { economic }^{\text {amod }}\end{array}$ & $\begin{array}{l}\text { civilzation, mythology, tradition, ruin, monument, trackway, tomb, antiquity, folklore, deity } \\
\text { blue }^{\text {amod }} \text {, black }^{\text {amod }}, \text { green }^{\text {amod }}, \text { dark }^{\text {amod }}, \text { onion, pepper, red, tomato, carrot, garlic } \\
\text { political }^{\text {amod }}, \text { societal } \\
\text { globalization, prosperity }\end{array}$ \\
\hline $\begin{array}{l}\text { government }^{\overline{\mathrm{dobj}}} \\
\text { problem }^{\overline{\mathrm{dobj}}} \\
\text { law }^{\overline{\mathrm{dobj}}}\end{array}$ & 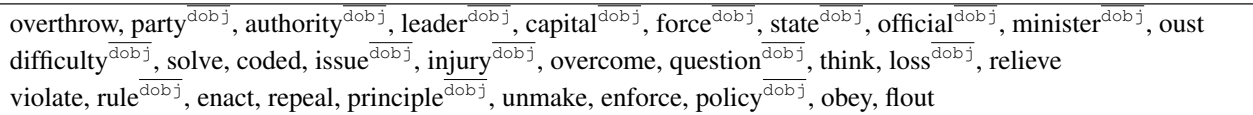 \\
\hline $\begin{array}{l}\text { researcher } \overline{\overline{\text { nubj }}} \\
\text { mother }^{\text {nsubj }} \\
\text { law }^{\text {nsubj }}\end{array}$ & 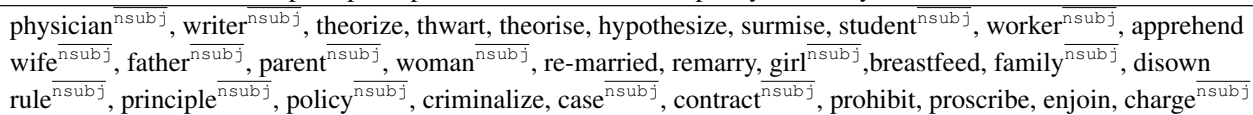 \\
\hline
\end{tabular}

Table 2: List of the 10 nearest neighbours of amod, $\overline{\mathrm{dob}_{j}}$ and $\overline{\mathrm{nsubj}}$ offset representations.

ing information, however with the risk of introducing some noise. The latter on the other hand can be used as a process of co-occurrence filtering, that is leveraging the enriched representations, while also sieving out the previously introduced noise.

\section{Experiments}

For our experiments we re-implemented the standard DI method of Kober et al. (2016) for a direct comparison. We built an order 2 APT space on the basis of the concatenation of ukWaC, Wackypedia and the BNC (Baroni et al., 2009), pre-parsed with the Malt parser (Nivre et al., 2006). We PPMI transformed the raw co-occurrence counts prior to composition, using a negative SPPMI shift of $\log 5$ (Levy and Goldberg, 2014b). We also experimented with composing normalised counts and applying the PPMI transformation after composition as done by Weeds et al. (2017), however found composing PPMI scores to work better for this task.

We evaluate our offset inference algorithm on two popular short phrase composition benchmarks by Mitchell and Lapata (2008) and Mitchell and Lapata (2010), henceforth ML08 and ML10 respectively. The ML08 dataset consists of 120 distinct verb-object (VO) pairs and the ML10 dataset contains 108 adjective-noun (AN), 108 noun-noun (NN) and 108 verb-object pairs. The goal is to compare a model's similarity estimates to human provided judgements. For both tasks, each phrase pair has been rated by multiple human annotators on a scale between 1 and 7, where 7 indicates maximum similarity. Comparison with human judgements is achieved by calculating Spearman's $\rho$ between the model's similarity estimates and the scores of each human annotator individually. We performed composition by intersection and tuned the number of neighbours by a grid search over $\{0$, $10,30,50,100,500,1000\}$ on the ML10 develop- ment set, selecting 10 neighbours for NNs, 100 for ANs and 50 for VOs for both DI algorithms. We calculate statistical significance using the method of Steiger (1980).

\section{Effect of the number of neighbours}

Figure 2 shows the effect of the number of neighbours for AN, NN and VO phrases, using offset inference, on the ML10 development set. Interestingly, NN compounds exhibit an early saturation effect, while VOs and ANs require more neighbours for optimal performance. One explanation for the observed behaviour is that up to some threshold, the neighbours being added contribute actually missing co-occurrence events, whereas past that threshold distributional inference degrades to just generic smoothing that is simply compensating for sparsity, but overwhelming the representations with non-plausible co-occurrence information. A similar effect has also been observed by Erk and Pado (2010) in an exemplarbased model.

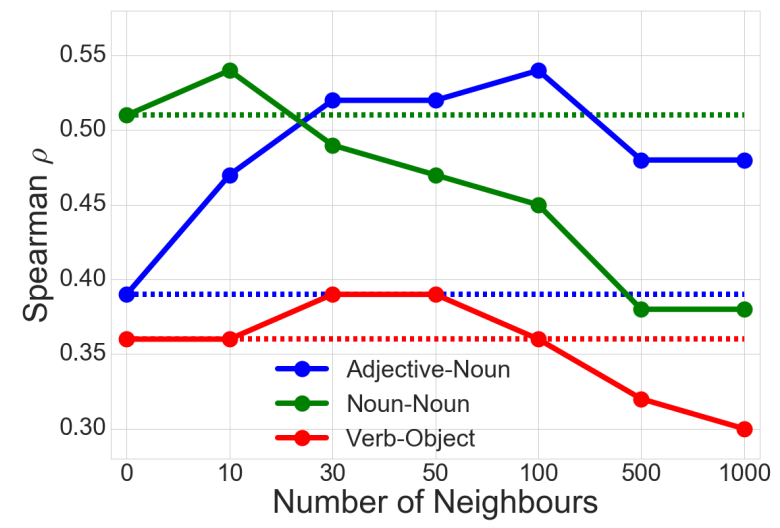

Figure 2: Effect of the number of neighbours on the ML10 development set.

\section{Results}

Table 3 shows that both forms of distributional inference significantly outperform a baseline without DI. On average, offset inference outperforms 
the method of Kober et al. (2016) by a statistically significant margin on both datasets.

\begin{tabular}{|c|c|c|c|c|c|}
\hline & \multicolumn{4}{|c|}{ ML10 } & ML08 \\
\hline APT configuration & $\mathbf{A N}$ & NN & VO & Avg & VO \\
\hline None & 0.35 & 0.50 & 0.39 & 0.41 & 0.22 \\
\hline Standard DI & $0.48^{\ddagger}$ & 0.51 & $0.43^{\ddagger}$ & $0.47^{\ddagger}$ & $0.29^{\ddagger}$ \\
\hline Offset Inference & $\mathbf{0 . 4 9} 9^{\ddagger}$ & 0.52 & $\mathbf{0 . 4 4}$ & $0.48^{* \ddagger}$ & $\mathbf{0 . 3 1} 1^{\dagger \ddagger}$ \\
\hline
\end{tabular}

Table 3: Comparison of DI algorithms. $\ddagger$ denotes statistical significance at $p<0.01$ in comparison to the method without DI, * denotes statistical significance at $p<0.01$ in comparison to standard DI and $\dagger$ denotes statistical significance at $p<0.05$ in comparison to standard DI.

Table 4 shows that offset inference substantially outperforms comparable sparse models by Dinu et al. (2013) on ML08, achieving a new state-ofthe-art, and matches the performance of the stateof-the-art neural network model of Hashimoto et al. (2014) on ML10, while being fully interpretable.

\begin{tabular}{l|c|c} 
Model & ML10 - Average & ML08 \\
\hline Our work & $\mathbf{0 . 4 8}$ & $\mathbf{0 . 3 1}$ \\
Blacoe and Lapata (2012) & 0.44 & - \\
Hashimoto et al. (2014) & $\mathbf{0 . 4 8}$ & - \\
Weir et al. (2016) & 0.43 & 0.26 \\
Dinu et al. (2013) & - & $0.23-0.26$ \\
Erk and Padó (2008) & - & 0.27
\end{tabular}

Table 4: Comparison with existing methods.

\section{Related Work}

Distributional inference has its roots in the work of Dagan et al. (1993, 1994), who aim to find probability estimates for unseen words in bigrams, and Schütze $(1992,1998)$ who leverages the distributional neighbourhood through clustering of contexts for word-sense discrimination. Recently Kober et al. (2016) revitalised the idea for compositional distributional semantic models.

Composition with distributional semantic models has become a popular research area in recent years. Simple, yet competitive methods, are based on pointwise vector addition or multiplication (Mitchell and Lapata, 2008, 2010). However, these approaches neglect the structure of the text defining composition as a commutative operation.

A number of approaches proposed in the literature attempt to overcome this shortcoming by introducing weighted additive variants (Guevara, 2010, 2011; Zanzotto et al., 2010). Another popular strand of work models semantic composition on the basis of ideas arising in formal semantics. Composition in such models is usually implemented as operations on higher-order tensors (Ba- roni and Zamparelli, 2010; Baroni et al., 2014; Coecke et al., 2011; Grefenstette et al., 2011; Grefenstette and Sadrzadeh, 2011; Grefenstette et al., 2013; Kartsaklis and Sadrzadeh, 2014; Paperno et al., 2014; Tian et al., 2016; Van de Cruys et al., 2013). Another widespread approach to semantic composition is to use neural networks (Bowman et al., 2016; Hashimoto et al., 2014; Hill et al., 2016; Mou et al., 2015; Socher et al., 2012, 2014; Wieting et al., 2015; Yu and Dredze, 2015), or convolutional tree kernels (Croce et al., 2011; Zanzotto and Dell'Arciprete, 2012; Annesi et al., 2014) as composition functions.

The above approaches are applied to untyped distributional vector space models where untyped models contrast with typed models (Baroni and Lenci, 2010) in terms of whether structural information is encoded in the representation as in the models of Erk and Padó (2008); Gamallo and Pereira-Fariña (2017); Levy and Goldberg (2014a); Padó and Lapata (2007); Thater et al. (2010, 2011); Weeds et al. (2014).

The perhaps most popular approach in the literature to evaluating compositional distributional semantic models is to compare human word and phrase similarity judgements with similarity estimates of composed meaning representations, under the assumption that better distributional representations will perform better at these tasks (Blacoe and Lapata, 2012; Dinu et al., 2013; Erk and Padó, 2008; Hashimoto et al., 2014; Hermann and Blunsom, 2013; Kiela et al., 2014; Turney, 2012).

\section{Conclusion}

In this paper we have introduced a novel form of distributional inference that generalises the method introduced by Kober et al. (2016). We have shown its effectiveness for semantic composition on two benchmark phrase similarity tasks where we achieved state-of-the-art performance while retaining the interpretability of our model. We have furthermore highlighted an interesting connection between distributional inference and distributional composition.

In future work we aim to apply our novel method to improve modelling selectional preferences, lexical inference, and scale up to longer phrases and full sentences. 


\section{References}

Paolo Annesi, Danilo Croce, and Roberto Basili. 2014. Semantic compositionality in tree kernels. In Proceedings of the 23rd ACM International Conference on Conference on Information and Knowledge Management. ACM, New York, NY, USA, CIKM '14, pages 1029-1038. https://doi.org/10.1145/2661829.2661955.

Marco Baroni, Raffaella Bernardi, and Roberto Zamparelli. 2014. Frege in space: A program for compositional distributional semantics. Linguistic Issues in Language Technology 9(6):5-110.

Marco Baroni, Silvia Bernardini, Adriano Ferraresi, and Eros Zanchetta. 2009. The wacky wide web: a collection of very large linguistically processed web-crawled corpora. Language Resources and Evaluation 43(3):209-226. https://doi.org/10.1007/s10579-009-9081-4.

Marco Baroni and Alessandro Lenci. 2010. Distributional memory: A general framework for corpus-based semantics. Computational Linguistics 36(4):673-721.

Marco Baroni and Roberto Zamparelli. 2010. Nouns are vectors, adjectives are matrices: Representing adjective-noun constructions in semantic space. In Proceedings of the 2010 Conference on Empirical Methods in Natural Language Processing. Association for Computational Linguistics, Cambridge, MA, pages 1183-1193. http://www.aclweb.org/anthology/D10-1115.

William Blacoe and Mirella Lapata. 2012. A comparison of vector-based representations for semantic composition. In Proceedings of the 2012 Joint Conference on Empirical Methods in Natural Language Processing and Computational Natural Language Learning. Association for Computational Linguistics, Jeju Island, Korea, pages 546556. http://www.aclweb.org/anthology/D12-1050.

Samuel R. Bowman, Jon Gauthier, Abhinav Rastogi, Raghav Gupta, Christopher D. Manning, and Christopher Potts. 2016. A fast unified model for parsing and sentence understanding. In Proceedings of the 54th Annual Meeting of the Association for Computational Linguistics (Volume 1: Long Papers). Association for Computational Linguistics, Berlin, Germany, pages 1466-1477. http://www.aclweb.org/anthology/P16-1139.

Bob Coecke, Mehrnoosh Sadrzadeh, and Stephen Clark. 2011. Mathematical foundations for a compositional distributed model of meaning. Linguistic Analysis 36(1-4):345-384.

Danilo Croce, Alessandro Moschitti, and Roberto Basili. 2011. Structured lexical similarity via convolution kernels on dependency trees. In Proceedings of the 2011 Conference on Empirical Methods in Natural Language Processing. Association for Computational Linguistics,
Edinburgh, Scotland, UK., pages 1034-1046. http://www.aclweb.org/anthology/D11-1096.

Ido Dagan, Shaul Marcus, and Shaul Markovitch. 1993. Contextual word similarity and estimation from sparse data. In Proceedings of the 31st Annual Meeting on Association for Computational Linguistics. Association for Computational Linguistics, Stroudsburg, PA, USA, ACL '93, pages 164-171. https://doi.org/10.3115/981574.981596.

Ido Dagan, Fernando Pereira, and Lillian Lee. 1994. Similarity-based estimation of word cooccurrence probabilities. In Proceedings of the 32nd Annual Meeting of the Association for Computational Linguistics. Association for Computational Linguistics, Las Cruces, New Mexico, USA, pages 272-278. https://doi.org/10.3115/981732.981770.

Georgiana Dinu, Nghia The Pham, and Marco Baroni. 2013. General estimation and evaluation of compositional distributional semantic models. In Proceedings of the Workshop on Continuous Vector Space Models and their Compositionality. Association for Computational Linguistics, Sofia, Bulgaria, pages 50-58. http://www.aclweb.org/anthology/W133206.

Katrin Erk and Sebastian Padó. 2008. A structured vector space model for word meaning in context. In Proceedings of the 2008 Conference on Empirical Methods in Natural Language Processing. Association for Computational Linguistics, Honolulu, Hawaii, pages 897-906. http://www.aclweb.org/anthology/D08-1094.

Katrin Erk and Sebastian Pado. 2010. Exemplarbased models for word meaning in context. In Proceedings of the ACL 2010 Conference Short Papers. Association for Computational Linguistics, Uppsala, Sweden, pages 92-97. http://www.aclweb.org/anthology/P10-2017.

Pablo Gamallo and Martín Pereira-Fariña. 2017. Compositional semantics using feature-based models from wordnet. In Proceedings of the 1st Workshop on Sense, Concept and Entity Representations and their Applications. Association for Computational Linguistics, Valencia, Spain, pages 1-11. http://www.aclweb.org/anthology/W17-1901.

E. Grefenstette, G. Dinu, Y. Zhang, M. Sadrzadeh, and M. Baroni. 2013. Multi-step regression learning for compositional distributional semantics. In Proceedings of the 10th International Conference on Computational Semantics (IWCS 2013) - Long Papers. Association for Computational Linguistics, Potsdam, Germany, pages 131142. http://www.aclweb.org/anthology/W13-0112.

Edward Grefenstette and Mehrnoosh Sadrzadeh. 2011. Experimental support for a categorical compositional distributional model of meaning. In Proceedings of the 2011 Conference on Empirical Methods in Natural Language Processing. Association for Computational Linguistics, 
Edinburgh, Scotland, UK., pages 1394-1404. http://www.aclweb.org/anthology/D11-1129.

Edward Grefenstette, Mehrnoosh Sadrzadeh, Stephen Clark, Bob Coecke, and Stephen Pulman. 2011. Concrete sentence spaces for compositional distributional models of meaning. Proceedings of the 9th International Conference on Computational Semantics (IWCS 2011) pages 125-134.

Emiliano Guevara. 2010. A regression model of adjective-noun compositionality in distributional semantics. In Proceedings of the 2010 Workshop on GEometrical Models of Natural Language Semantics. Association for Computational Linguistics, Uppsala, Sweden, pages 33-37. http://www.aclweb.org/anthology/W10-2805.

Emiliano Guevara. 2011. Computing semantic compositionality in distributional semantics. In Proceedings of the Ninth International Conference on Computational Semantics. Association for Computational Linguistics, Stroudsburg, PA, USA, IWCS '11, pages 135-144.

Kazuma Hashimoto, Pontus Stenetorp, Makoto Miwa, and Yoshimasa Tsuruoka. 2014. Jointly learning word representations and composition functions using predicate-argument structures. In Proceedings of the 2014 Conference on Empirical Methods in Natural Language Processing (EMNLP). Association for Computational Linguistics, Doha, Qatar, pages 1544-1555. http://www.aclweb.org/anthology/D14-1163.

Karl Moritz Hermann and Phil Blunsom. 2013. The role of syntax in vector space models of compositional semantics. In Proceedings of the 51st Annual Meeting of the Association for Computational Linguistics (Volume 1: Long Papers). Association for Computational Linguistics, Sofia, Bulgaria, pages 894-904. http://www.aclweb.org/anthology/P131088.

Felix Hill, KyungHyun Cho, Anna Korhonen, and Yoshua Bengio. 2016. Learning to understand phrases by embedding the dictionary. Transactions of the Association for Computational Linguistics 4:17-30. http://www.aclweb.org/anthology/Q/Q16/Q161002.pdf.

Dimitri Kartsaklis and Mehrnoosh Sadrzadeh. 2014. A study of entanglement in a categorical framework of natural language. In Proceedings of the 11th Workshop on Quantum Physics and Logic (QPL).

Douwe Kiela, Felix Hill, Anna Korhonen, and Stephen Clark. 2014. Improving multi-modal representations using image dispersion: Why less is sometimes more. In Proceedings of the 52nd Annual Meeting of the Association for Computational Linguistics (Volume 2: Short Papers). Association for Computational Linguistics, Baltimore, Maryland, pages 835841. http://www.aclweb.org/anthology/P14-2135.
Thomas Kober, Julie Weeds, Jeremy Reffin, and David Weir. 2016. Improving sparse word representations with distributional inference for semantic composition. In Proceedings of the 2016 Conference on Empirical Methods in Natural Language Processing. Association for Computational Linguistics, Austin, Texas, pages 1691-1702. https://aclweb.org/anthology/D16-1175.

Omer Levy and Yoav Goldberg. 2014a. Dependencybased word embeddings. In Proceedings of the 52nd Annual Meeting of the Association for Computational Linguistics (Volume 2: Short Papers). Association for Computational Linguistics, Baltimore, Maryland, pages 302-308. http://www.aclweb.org/anthology/P14-2050.

Omer Levy and Yoav Goldberg. 2014b. Neural word embedding as implicit matrix factorization. In Z. Ghahramani, M. Welling, C. Cortes, N.D. Lawrence, and K.Q. Weinberger, editors, Advances in Neural Information Processing Systems 27, Curran Associates, Inc., pages 2177-2185.

Jeff Mitchell and Mirella Lapata. 2008. Vector-based models of semantic composition. In Proceedings of ACL-08: HLT. Association for Computational Linguistics, Columbus, Ohio, pages 236-244. http://www.aclweb.org/anthology/P/P08/P08-1028.

Jeff Mitchell and Mirella Lapata. 2010. Composition in distributional models of semantics. Cognitive Science 34(8):1388-1429. https://doi.org/10.1111/j.1551-6709.2010.01106.x.

Lili Mou, Hao Peng, Ge Li, Yan Xu, Lu Zhang, and Zhi Jin. 2015. Discriminative neural sentence modeling by tree-based convolution. In Proceedings of the 2015 Conference on Empirical Methods in Natural Language Processing. Association for Computational Linguistics, Lisbon, Portugal, pages 23152325. http://aclweb.org/anthology/D15-1279.

Joakim Nivre, Johan Hall, and Jens Nilsson. 2006. Maltparser: A data-driven parser-generator for dependency parsing. Technical report, Växjö University.

Sebastian Padó and Mirella Lapata. 2007. Dependency-based construction of semantic space models. Computational Linguistics 33(2):161-199. https://doi.org/10.1162/coli.2007.33.2.161.

Denis Paperno, Nghia The Pham, and Marco Baroni. 2014. A practical and linguistically-motivated approach to compositional distributional semantics. In Proceedings of the 52nd Annual Meeting of the Association for Computational Linguistics (Volume 1: Long Papers). Association for Computational Linguistics, Baltimore, Maryland, pages 9099. http://www.aclweb.org/anthology/P14-1009.

Hinrich Schütze. 1992. Dimensions of meaning. In Proceedings of ACM/IEEE Conference on Supercomputing. IEEE Computer Society Press, pages 787-796. http://dl.acm.org/citation.cfm?id=147877.148132. 
Hinrich Schütze. 1998. Automatic word sense discrimination. Computational Linguistics 24(1):97-123.

Richard Socher, Brody Huval, Christopher D. Manning, and Andrew Y. Ng. 2012. Semantic compositionality through recursive matrix-vector spaces. In Proceedings of the 2012 Joint Conference on Empirical Methods in Natural Language Processing and Computational Natural Language Learning. Association for Computational Linguistics, Jeju Island, Korea, pages 1201-1211. http://www.aclweb.org/anthology/D12-1110.

Richard Socher, Andrej Karpathy, Quoc Le, Christopher Manning, and Andrew Ng. 2014. Grounded compositional semantics for finding and describing images with sentences. Transactions of the Association for Computational Linguistics 2:207-218.

James H Steiger. 1980. Tests for comparing elements of a correlation matrix. Psychological Bulletin 87(2):245.

Stefan Thater, Hagen Fürstenau, and Manfred Pinkal. 2010. Contextualizing semantic representations using syntactically enriched vector models. In Proceedings of the 48th Annual Meeting of the Association for Computational Linguistics. Association for Computational Linguistics, Uppsala, Sweden, pages 948-957. http://www.aclweb.org/anthology/P101097.

Stefan Thater, Hagen Fürstenau, and Manfred Pinkal. 2011. Word meaning in context: A simple and effective vector model. In Proceedings of 5th International Joint Conference on Natural Language Processing. Asian Federation of Natural Language Processing, Chiang Mai, Thailand, pages 1134-1143. http://www.aclweb.org/anthology/I11-1127.

Ran Tian, Naoaki Okazaki, and Kentaro Inui. 2016. Learning semantically and additively compositional distributional representations. In Proceedings of the 54th Annual Meeting of the Association for Computational Linguistics (Volume 1: Long Papers). Association for Computational Linguistics, Berlin, Germany, pages 1277-1287. http://www.aclweb.org/anthology/P16-1121.

Peter D. Turney. 2012. Domain and function: A dual-space model of semantic relations and compositions. Journal of Artificial Intelligence Research 44(1):533-585.

Tim Van de Cruys, Thierry Poibeau, and Anna Korhonen. 2013. A tensor-based factorization model of semantic compositionality. In Proceedings of the 2013 Conference of the North American Chapter of the Association for Computational Linguistics: Human Language Technologies. Association for Computational Linguistics, Atlanta, Georgia, pages 11421151. http://www.aclweb.org/anthology/N13-1134.

Julie Weeds, Thomas Kober, Jeremy Reffin, and David Weir. 2017. When a red herring in not a red herring: Using compositional methods to detect non-compositional phrases. In Proceedings of the 15th Conference of the European Chapter of the Association for Computational Linguistics: Volume 2, Short Papers. Association for Computational Linguistics, Valencia, Spain, pages 529-534. http://www.aclweb.org/anthology/E17-2085.

Julie Weeds, David Weir, and Jeremy Reffin. 2014. Distributional composition using higher-order dependency vectors. In Proceedings of the 2nd Workshop on Continuous Vector Space Models and their Compositionality (CVSC). Association for Computational Linguistics, Gothenburg, Sweden, pages 11-20. http://www.aclweb.org/anthology/W141502.

David Weir, Julie Weeds, Jeremy Reffin, and Thomas Kober. 2016. Aligning packed dependency trees: a theory of composition for distributional semantics. Computational Linguistics, special issue on Formal Distributional Semantics 42(4):727-761.

John Wieting, Mohit Bansal, Kevin Gimpel, and Karen Livescu. 2015. From paraphrase database to compositional paraphrase model and back. Transactions of the Association for Computational Linguistics 3:345-358. http://www.aclweb.org/anthology/Q/Q15/Q151025.pdf.

Mo Yu and Mark Dredze. 2015. Learning composition models for phrase embeddings. Transactions of the Association for Computational Linguistics 3:227242. http://aclweb.org/anthology/Q/Q15/Q151017.pdf

Fabio Massimo Zanzotto and Lorenzo Dell' Arciprete. 2012. Distributed tree kernels. In John Langford and Joelle Pineau, editors, Proceedings of the 29th International Conference on Machine Learning (ICML-12). Omnipress, New York, NY, USA, ICML '12, pages 193-200.

Fabio Massimo Zanzotto, Ioannis Korkontzelos, Francesca Fallucchi, and Suresh Manandhar. 2010. Estimating linear models for compositional distributional semantics. In Proceedings of Coling. pages 1263-1271. http://www.aclweb.org/anthology/C101142. 